\section{DENTAL SEPSIS.}

By J. G. Torner, F.R.C.S.

(Continued from page 1248.)

\section{ProphyLAXIs.}

The answer to the third question is best sought for by an inquiry as to causation, and every tooth-bearing anima may be studied with advantage. The mouth is obviously fouled from without. The infant's mouth is as early infected as any other of its outward openings, but, in general, the capacity for injury depends on stagnation. The germs then have time to establish themselves. If sticky food be added they are provided with unlimited culture media. Such haphazard conditions may not suit all germs, but in fact do suit many, and experience tells us that the net result is unfarourable to human happiness.

Food.

What food, then, is sticky? Above all, fine-ground flour. Our inquiry may run on these broad lines: What is the relation of dental sepsis to the occurrence of micro organisms in the mouth and to the eating of fine-ground flour? Shortly, wo mase the relation of dental sepsis to germ-carbohydate atanngtion.

I have examined fish, and have never yet found any evidence of dental sepsis among them. I made films of scrapings from the mouth of a large number of deep-sea tish very shortly after capture, and could demonstrate no micro-organisms. There was also not evien a shred of seaweed in their mouths. In the absence of all stagnation there was no dental sepsis. . In the large collection of skulls of wild carnivora (tigers, etc.) at the South Kensington Museum I found no post-mortem evidence of dental sepsis other than abscess following fractured tooth, except in the case of an old hyena from Somaliland, presented by Dr. Drake-Brockman. The sepsis in this case probably followed some gross injury to the gum by coarse food. Among domesticated carnivora (dogs) evidences of dental sepsis, especially foul teeth, and alveolar disease are common. Bread, cake, and biscuits are part of the daily food of the domestic dog.

Examination of skulls of wild monkeys at the South Kensington Museum showed 0.53 per cent. of teeth carious, and 5.31 per cent. of skulls containing carious teeth. 'The total of skulls was 433, containing 12,249 teeth; 58 teeth had been lost ante mortem. The skulls of 72 monkeys which had died at the London Zoological Society's Gardens gave 16.66 per cent. containing carious teeth, and 2.001 per cent. of teeth carious. I do not know much of the feeding habits of wild monkeys, but it seems probable that nuts or plantains may be responsible for the occurrence of dental caries. Domesticated monkeys are largely fed on bread. Pyorrhoea alveolaris occurs among wild monkeys, but only in its localized form. The bone is found destroyed between two teeth, the rest of the mouth being sound. In this pocket coarse spikes of vegetable origin are found, and the conclusion is irresistible that the disease was the result of gross trauma while eating.

The skulls of captive monkeys, on the other hand, show widespread alveolar changes comparable to what is found in human beings. Among wild monkeys, again, tartar occurs in small amounts, but among the captive monkeys in very considerable quantity.

In skulls of wild grass-eating animals I have seen no post-mortem evidence of dental sepsis. Films from the mouths of domesticated cattle show large numbers of bacteria, but, except mycotic infections, I know of no resulting disease condition. This mycotic infection is of the same nature as the localized pyorrhoea alveolaris of wild monkeys. To this category belongs also the alveolar disease of the domestic horse; in these animals spikes of chaffed hay get speared into the gum in mastication, causing localized but not generalized disease.

Among humankind we of to-day are the most notable consumers of sticky carbohydrates, and in the matter of dental sepsis we hold an unenviable pre-eminence. Practically 100 per cent. of the population of Great Britain suffer from dëntal caries. IPractically 100 per cènt. are affected by marginal gingivitis or its later developments. Extensive deposit of tartar is to be found in almost every mouth. The number of teeth lost ante mortem is beyond counting: Before the introduction of fine milling things vere not so bad. Of 19 skulls from a cemetery disused since 1840, carious teeth were present in 7 and 5 showed post-mortem signs of pyorrhoea alveolaris. Of 288 teeth present, 15 showed caries and 28 teeth had been lost ante mortem.

Of $\mathbf{4 0}$ skulls from a cemetery disused since 1830 carious teeth were present in 14 and 14 showed post-mortem signs of pyorrhoea alveolaris ( 7 of these are queried-that is, were very slight); of 567 teeth, 55 were carious; excluding old skulls, 17 teeth had been lost ante mortem; 17 skulls showed deposits of cervical tartar. In many of the skulls the evidences of pyorrhoea were limited to the molars, eren to only one or two of them. Of 791 teeth found in maxillas and mandibles representing 90 skulls believed to be remains of the plague of London and now in the possession of University College, 19.5 per cent. were carious, and 35.5 per cent. of skulls contained carious teeth ; 439 teeth had been lost ante mortem. Most of the jaws showed some signs of alveolar destruction (pyorrhoea alveolaris) but only few well marked cases were found. Cervical tartar was also present in most.

Of 75 crania from the collection in the crypt of Rothwell Church, dating back at least 300 years, 16 contained carious. teeth; 31 out of 440 teeth present were carious (7.04 per cent.), and 30 teeth had been lost ante mortem; 25 skulls. showed evidence of pyorrhoea alveolaris. This was found among the molars only, and was bad in only 2 cases. Cervical tartar was present in 35 skulls.

The cereal food of all these groups. was, as compared with ours of to-day, coarsely ground, and the local results of dental sepsis-decay of teeth and peripheral alveolar caries (pyorrhoea alveolaris)-are correspondingly less. The tartar present is mostly limited to a small ring at the neck, and probably represents the accumulation of a lifetime.

Of 435 teeth which had fallen from the sockets of ancient Egyptian skulls of the twenty-fifth to thirtieth dynasties, 16.78 per cent. were carious. Many of the cavities were very small, and woula probably not have been found but for the minute examination permitted by their having fallen out. Only one tòth, a molar, showed evidence of pyorrhoea alveolaris. Professor Karl Pearson's assistants kindly charted 104 maxillas of these dynasties : 42 of these contained carious teeth; 738 teeth were present, with a percentage of 9.75 carious; 133 teeth had been lost ante mortem. The cereal food of these people was coarsely stone-ground.

Thus in three important points of dental sepsis the coarser cereal feeders compare favourably with the fine. ground feeders. There is one point, however, in which they compare less favourably-namely, in the occurrence of dental abscess. Dental abscess was common as a result of exposure and death of the pulp consequent on excessive grinding down of the teeth. This was commonest in the case of the first permanent molars, and accounts largely for the teeth lost ante mortem.

The relation between dental sepsis and sticky vegetable food is well shown by the state of many of the jaws of the Polynesian skulls preserved in the museum of the Royal College of Surgeons. In many of the islands cocoanut is a staple food, and is often so prepared as to make a very sticky food. The jaws above alluded to show extensive cervical caries, and also alveolar destruction (pyorrhoea alveolaris) especially among the molars. In several of the skulls this condition is accompanied by temporo-mandibular arthritis.

The Prevention of Dental Sepsis.

The causation of dental sepsis in man may be' sum. marized thus: Germs are present in every human mouth. If they are allowed to stagnate they will multiply and produce evil results in the absence of any other factor. In this respect we may compare the sclid stagnation mentioned above and the evidences of pyorrhoea alveolaris in the comparatively clean-eating early English skulls. If another factor-sticky, starchy food (fine-ground flour in particular)-be added, dental caries, pyorrhoea alveolaris, and food decomposition follow, increasing in extent with the stickiness of the food. The deposition of tartar is dependent on stagnation, and is found to some extent in most adult human skulls, but is less extensive where coarser cereals were used, and increases with the use of sticky bread-food.

In the light of these considerations, prevention of dental sepsis may be hoped for (1) by reverting to the use of 
coarse-ground flour; (2) by accepting the present flour, * but insisting on actual cleansing of the teeth at least once laily - that is, by prevention of stagnation. What passes jor cleansing is but an excessive rubbing of the larger, but less important, part of the visible surfaces of the ten anterior teeth. The necks and the abutting surfaces and the hindmost teeth are not touched, and it is exactly in these danger areas that pyorrhoea alveolaris, and the greater part of dental caries, arises.

Sepsis in the mouth depends entirely on the presence of teeth, and if these be kept properly clean dental sepsis and its sequellae are impossible.

Method of Cleansing the Teeth.

Cleansing of teeth is difficult. Some, indeed, deny its utility. They claim to have discovered a method of feeding which banishes dental caries. Were this claim a proven fact, there still remain alveolar infections and decomposition of stagnant débris, and examination of the "uncleansed" mouth shows that these processes are in full swing. If only for the comfort of one's neighbour the teeth should be cleansed.

Unfortunately, the shapes and arrangement of the teeth are such as to make stagnation of sticky material inevitable unless artificial means be adopted for its prevention, and the problem is to make the best use of the artificial means of cleansing at our disposal. These are toothbrush, washes, powders, and pastes, and silk (or other) thread.

The Toothbrush.

The brush should be small. The large sizes are an absurdity-there is no room in the mouth to use them.

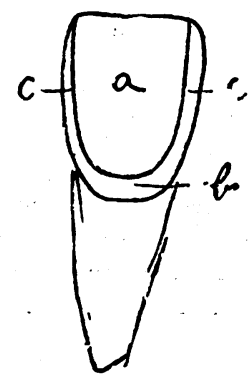

Fig. 1.-Diagram of an upper central incisor, to show the large central easily-cleansed area of $a$, and the small, less easily cleansed, and therefore neglected areas, $b$ and $c, c . \quad B y$ referring to the diagrans of vertical and transverse sections it will be seen areas.

Vigorous movement can be imparted to a small brush. The parts of the teeth accessible to the brush are the vestibular (outer), coronal (eating), and lingual (inner) surfaces. For cleansing purposes the outer and inner

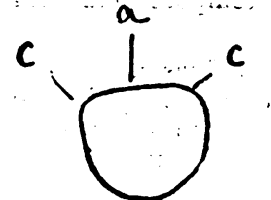

Fig. 3.-Transverse section of same towards the neck. $a$. Large easily cleaned area; $c, c$, small lateral receding areas be. coming continuous with

surfaces must be thought of as having four distinct areas. First, the-large, easily-cleansed central portion (a); second, the small receding cervical portion $(b)$; third and fourth, the two lateral parts $(c, c)$ where the outer (or inner) surface becomes continuous with the sides of the tooth. These, again, are receding areas. The large area " $a$ " of the outcr surfaces can be cleansed easily by brushing in a horizontal direction with the bristles normal to its surface, but the mistake is usually made of brushing only the more anterior teeth. The back teeth are missed because the curve of the dental arch is not realized, and the brush is actually directed away from them, or because the brush is too thick to pass between them and the

* Stale bread made of fine flour eats much cleaner than new bread. ascending ramus. To remedy this latter point we want brushes with shorter bristles. To overcome the former the handle of the brush should be carried outwards, stretching the corner of the month, while the brush itself is kept pressed against the teeth Sometimes it is

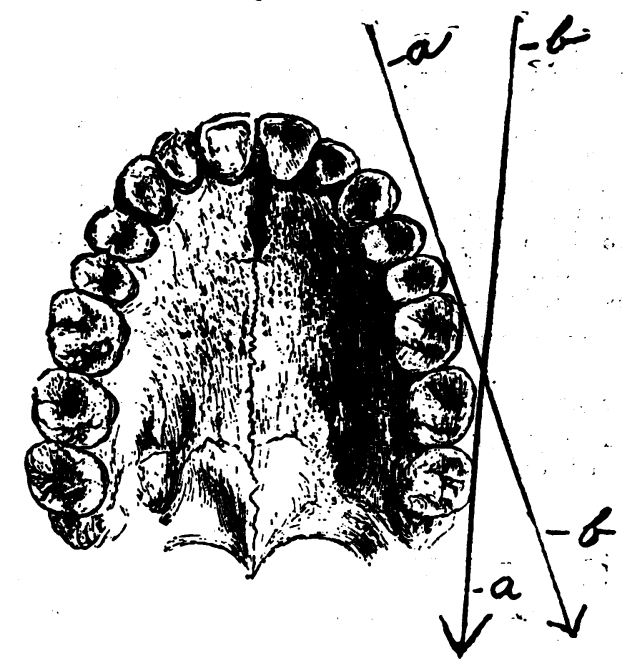

Fig. 4. - Showing how the brush misses the outside of the upper molars on account of the horse-shoe shape of the normal dental arch. The arrow $a$ shows the wrong direction of the brush, $b$ the right direction when the angle of the month is drawn aside. (Reproduced, by kind permission of Messrs. J. and A. Churchill,

possible by thus drawing aside the angle of the mouth to get to the back of the last tooth standing.

To clean the cervical area $(b)$ the brush is to be usad again in a horizontal direction, but is to be tilted till $t$

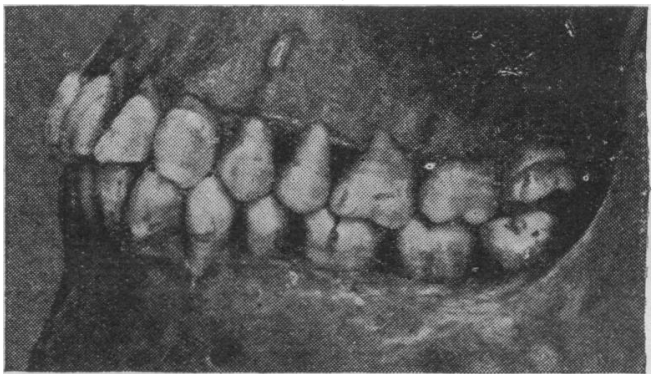

Fig. 5.-Showing that the last upper molar is of ten at a higher level than the others, and hence escapes the brush. As each or the two more anterior molars erupts, it occupies a similar position and so for some time runs a similar risk. The sgure also shows how little room there is between the ascending ramus and the last molars,

necks of the upper teeth are brushed only by the upper edge of the brush, and those of the lower only by the lower edge. In these positions the bristles are at right angles to the small cervical area (as shown by the tangent

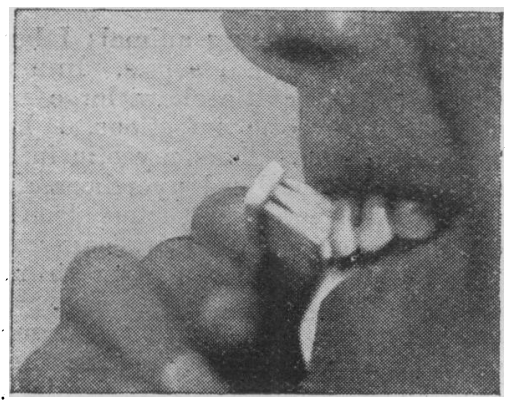

Fig. 6.-End-on view of the brush tilted to clean the necks of the upper.

and normal in Fig. 2) and more or less parallel to the gum, which is thus protected from injury.

To reach: the: lateral receding parts the toeth should be closed as for nibbling, or biting a thread, and the brush 
used in a vertical direction though still held with its long axis horizontal. If the brush is ridged transversely, the longer bristles will have an opportunity of getting between the teeth.

In brushing the coronal surfaces it is again the back teeth which are apt to be missed, especially when, as

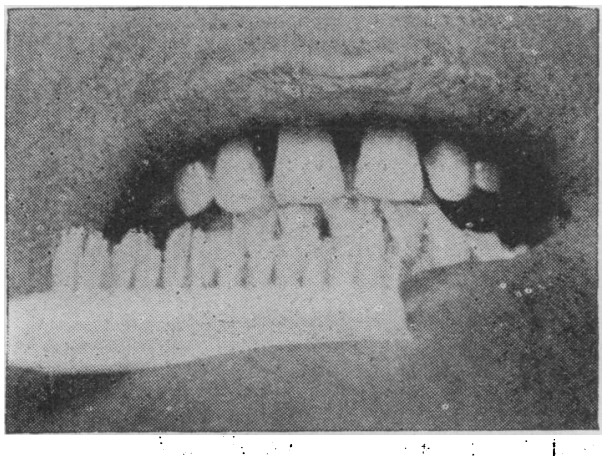

Fig. 7.-A front view of the brush tilted to clean the necks of the lower teeth.

often happens, the last upper molar has not come down into horizontal alignment.

'The inner surfaces of the teeth are obviously less accessible to cleaning, but no inconsiderable use may be made of the brush, and the larger and better formed the dental arch the better chance will there be of cleansing.

The brush again is to be held with its long axis as near as may be horizontal, and its bristles as near as possible at right angles to the tooth surfaces; it will generally be

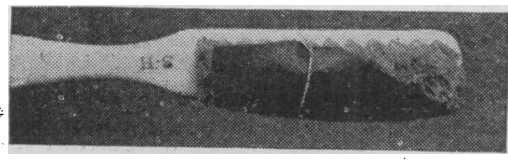

Fig. 8. The uncovered triangular part is the only part used when cleaning the inner sides of the teeth.

found impracticable to tilt the brush and so reach the necks of the teeth, but the tongue is by no means a despicable agent of cleanliness in this direction. Only an elongated triangular part of the end of the brush can be effective, but this can be moved to and fro horizontally in a very effective manner. In dealing with the front teeth the brush may be held firmly pressed against the teeth, while the head is rigorously nodded from side to side. The inner sides of the cheek teeth are dealt with by using

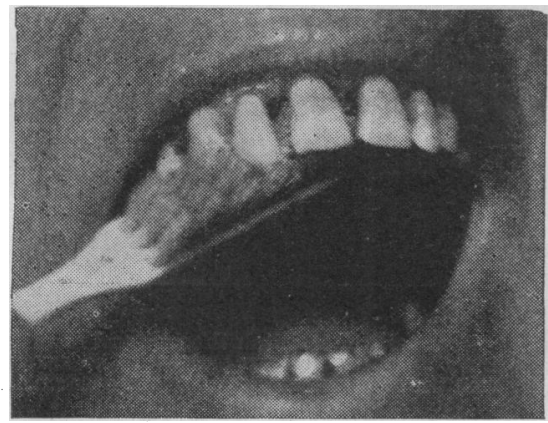

Fig. 9.-Showing the method of introducing the brush to clean the inner surfaces of the upper front teeth. Only a small triangular portion of the brush is in use. The bristles are as near as possible at right angles to the surfaces to be cleaned.

the same trianguiar part, moving the brush to and fro in the horizontal direction. On the inuer sides of the teeth very little vertical movement can be effected, and the parts marked $c c$ in the diagram must be cleaned by means of silk.

By careful attention it is possible to cultivate a special sense of the position of the bristles in relation to the gum edge.
The brush should be small, ridged transversely, and the bristles short and stiff rather than long and soft. A little observation will show that, though the back of the brush move freely, the ends of long soft bristles remain stationary; they merely bend to and fro in their length.

After use the brush should be well washed in a stream of cold water (under the tap) and dried, or, as Elander recommends, washed and left to soak for some hours in a weak solution of solveol, and then again washed in water and dried. Solveol is cheap, and does not injure the bristles.

Tooth Washes, Powders, and Pastes.

If the toothbrush be not properly used, the particular wash, powder, or paste matters not one iota.

Teeth are covered by a layer of mucus, to which, wherever stagnation is possible, germs and food débris are added. Mere washing the mouth with antiseptic solutions is of as much use as water to clean a duck's back, and the laboratory proof of their efficacy is beside the mark. Unless the mucus and débris be rubbed away, antiseptics cannot reach their objective, and when this is done there is not much left for antiseptics to achieve. Myself, I ad wise an acid tooth-wash to dissolve or assist in breaking up the mucus coat, and a tooth-powder occasionally, and to those who can afford it I recommend to finish their meals with an acid fruit while yet hungry.

Use of Silk Thread.

The use of silk thread is decried by many because of the fear of injuring the gums. Properly used there is no injury. It is our only means of cleaning betweer the teeth, and with our present food interstitial trouble (caries, tartar, gingivitis, and its sequelae) is bound to supervene in the absence of interstitial cleaning.

The silk should be waxed to allow of its slipping easily between closely set teeth, and it may be dipped in an antiseptic solution in the hope of carrying some up with it. Unwaxed thread will carry more, but unwaxed thread will not slip up between closely set teeth.

To explain the use of silk, the terms mesial and distal must be used. Imagine the teeth of either jaw spread out into a straight row, each preserving its relative position to its neighbour. Between the two central incisors draw a vertical line. Then the side of each tooth nearer this line is its mesial surface, that further away is its distal surface. The mesial and distal surface of each tooth has to be cleaned. These surfaces are not simple flat surfaces, but recede at the gum edge, where the diameter of the neck is less than that of the crown, and are rounded off

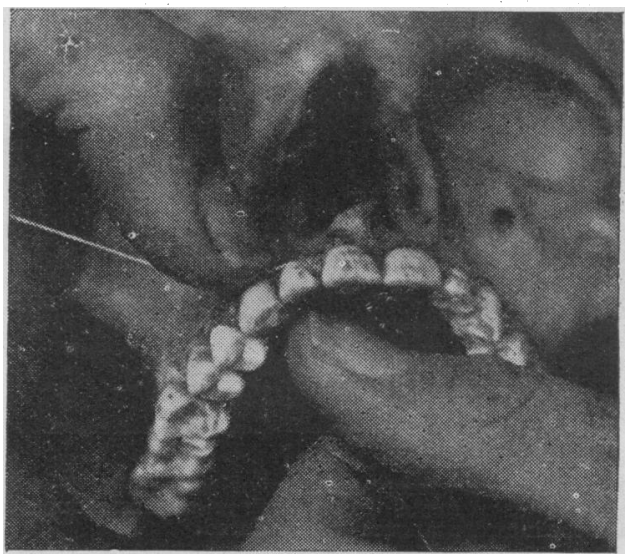

Fig. 10.-Showing the right method of carrying the thread well under the edge of the gum and round the tooth, and of making pressure against the surface to be cleaned.

where they become continuous with the areas marked c $c$ in the first diagram.

In using the silk keep the thread always in contact with the surface to be cleaned and with that alone. Pass it gently up (or down) lightly in contact with that surface, as far under the gum as it will go and encircling as much of the tooth as possible, so as to include the areas marked $c c$. Press firmly against the surface to be cleaned, and still keeping up the pressure and encircling the tooth, draw the thread 
back. If the thread be passed up gently, kept always in contact with the one tooth surface, and only the vertical movement used, no injury will be done to the gum. Injury is done (1) by passing the thread up roughly (if the teeth are closely pressed together short sideways sawing movements can be used to pass the thread, so that there is little momentum when it clears the tight area); (2) by sawing to and fro sideways when the silk is up (or down) (this is a movement of little value in cleaning) ; (3) by not keeping against one tooth only (the thread is either passed

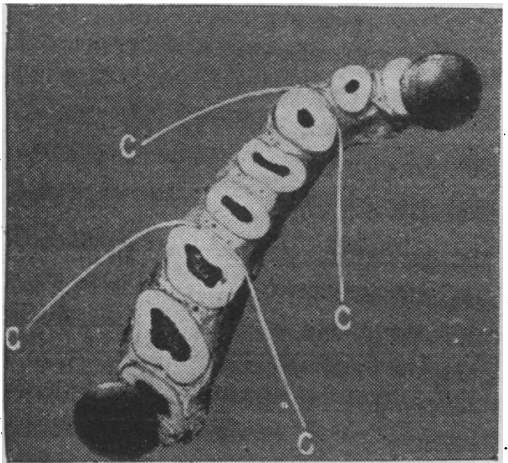

Fig. 11.-Shows the thread " as far under the edge of the gum as it will go, and as far round the tooth as it will go." If an attemp from the position shown directly to the neighbouring tooth th interdental gum-pad will be injured. (The teeth have been ground level with the alveolar bone.)

up against, say, the mesial surface of one tooth till it is under the gum, and then carried across to the distal surface of its neighbour, nipping the gum on its way, or it is carried up diagonally, touching an edge of the mesial surface of one tooth and an edge of the distal surface of its neighbour, in which :case the gum receives the impact of the thread; and is also nipped if the thread be approximated to either tooth). These three points, together with the loss of sense of direction noted below, explain both the failure to clean by: use: of silk thread and the injury whioh causes its condemnation. :...

In using the silk the ends must be firmly held. This may be done with the second, third, and fourth fingers.

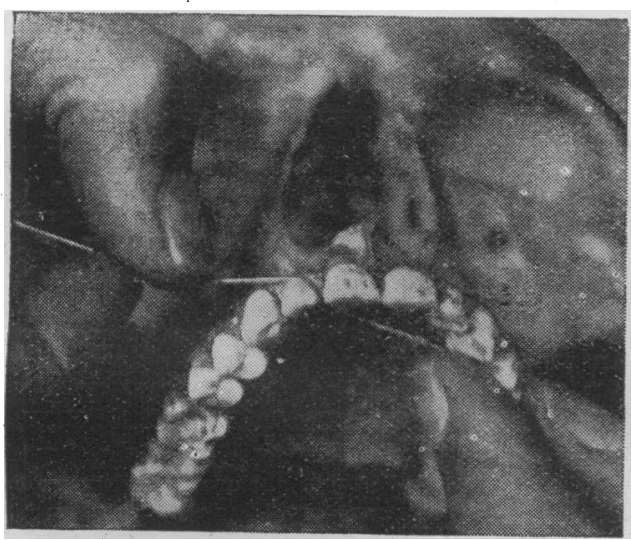

Fig. 12.-Showing a wrong method of using the silk. The thread is touching a distal angle of one tooth and a mesial angle of the next, and the interdental gum-pad is injured.

If a reel of silk be used, the reel may be held by these three fingers against the palm of one hand, and the free end of the thread twisted round the same fingers of the other hand till it cannot slip. 'Two hitches will suffice for this. The advantage of using a simple reel of silk is that a fresh inch can be unwound for each tooth. The thumb and forefinger are free to manipulate the silk. Thero should be only enough silk between the two hands to allow of a free piece of 1 in. or less between the two thumbs (or forefingers when these are in the correct position of use), the idea being that both the inner and outer sides of the tooth to be cleaned can be felt by the manipulating fingers (or thumbs) and the actual position of the thread thus ascertained. Holding the silk tense between the two hands, stretch the two fingers (or thumbs) to their full length, and place them against the thread so that it lies along the middle line of their tips and leaves a free inch or less between them. Keep the unoccupied fingers or thumbs clear of the thread; at first the natural tendency is to try to hold the thread between the forefinger and thumb of each hand, but there is no room in the mouth for both fingers and thumbs, nor can the thread be held firmly in this way.

The thumbs are used for cleaning the upper teeth, the

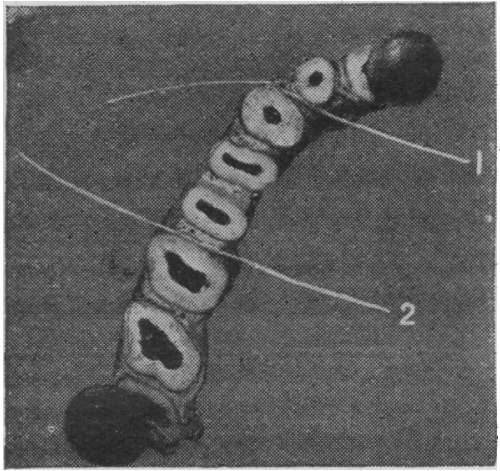

Fig. 13.-Showing two wrong positions: (1) where the thread is touching a distal angle of one tooth and a mesial angle of its thread on either side of a molar is carried =upwaids in to the palate and vestibule instead of backwards round the tooth.

fingers for the lower. Beginning now at the distal surface of the last upper molar, pass one thumb back into the mouth inside the cheek, but outside the teeth, and the other inside the teeth, keeping the free inch of silk tense in the transverse direction; - the sense of direction is readily lost, and most people have changed to the anteroposterior by the time the thumbs are in the mouth. Witl the thumbs (or thumbnails) feel the sides of the tooth to be cleaned, and so guide the thread up behind the tooth as far as it can go, keeping it lightly against the surface to be cleaned, press the silk firmly against the tooth by drawing both hands forward (in the mesial direetion)-aiac so pressing draw the silk down. In a similar. way find the mesial surface of the last tooth and adjust the thread, but now the hands must make pressure backwards (in the distal direction); and again the sense of direction is often at fault, and most people begin by making pressure upwards (see Fig. 13). When the front teeth are reached,

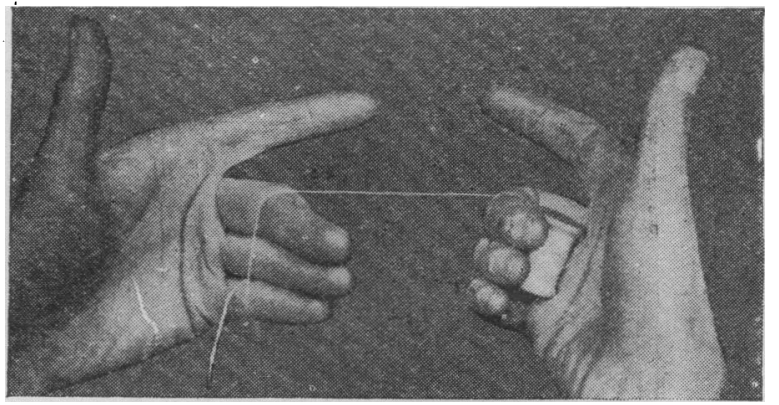

Fig. 14.-To hold the silk fast, hitch the free end round the little anger, and bring the other part up and over the second finger.

it is obvious that the direction of the free piece of silk must be antero-posterior, but the idea remains the same: with one thumb inside the teeth and the other outside, press mesially to clean a distal surface and distally to clean a mesial surface, embrace as much of the tooth as possible, and keep only to the surface to be cleaned.

The description is necessarily lengthy, and the learning undoubtedly difficult, but once learnt the process occupies but a minute or two, and the benefits are well worth the initial trouble.

I adrocate teaching children the use of silk as soon as they get any permanent teeth. Inflammation of the interstitial gum pad and interstitial decay of the teeth are to 
be found in almost every child, and only such means as above described can reach the seat of injury.

Where there is room a small-nozzled syringe may be used, but at best only the gross débris will be disturbed; the sticky layers on the teeth and the less roomy pockets (a syringe can only be useful when destruction of tissue has occurred) will be untouched. Under these conditions steel probes of varying fineness will bo found more useful. A wisp of cotton-wool is rolled round the end, dipped in an acid tooth-wash, and with it the sides of the teeth inaccessible to the toothbrush can be vigorously rubbed. To make effective use of the probe the patient must learn the varying sliapes of the teeth and appreciate their curves and complexities. As the roots become more exposed, the difficulties of cleaning are increased.

Some recommend the use of pledgets of cotton-wool held between the beaks of dental tiveezers for the treatment of the marginal gingivitis of children." Tweezer beaks and pledgets seem to ne too coärse to reach the small danger area - they bridge it over -and one can sometimes see that the effort to clean between the teeth has only resulted in forcing in more débris.

\section{General Directions.}

Teeth should be cleaned the last thing at night, and nothing caten thereafter till the next day. They should

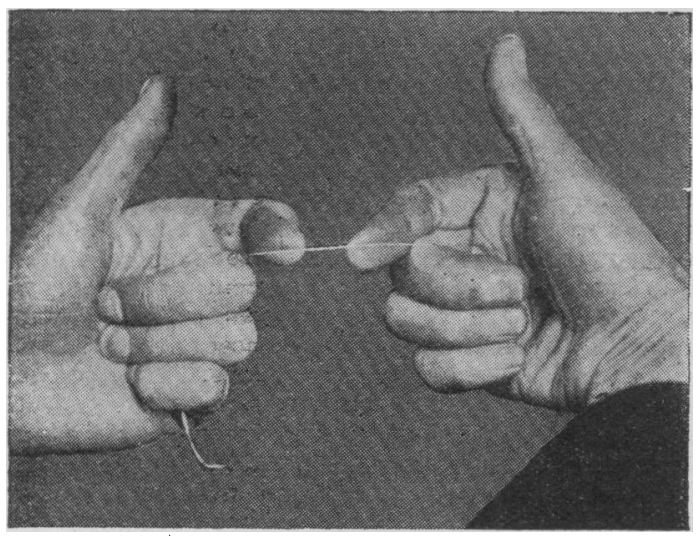

Fig. 15.-Showing how to manipulate the silk thread with the forefingers. The free end is now held by the fingers grasped dgainst the palm.

be examined in a mirror before and after cleaning to make sure of the effectiveness of the process. A little experience will show that the large area marked $a$ in the first diagram is the part which gets most of the rubbing and that the receding parts $b, c c$, are practically left untouched. Under these conditions cleaning the teeth a dozen times a day is of no use whatever in the prevention of sepsis; the part cleaned is exactly the part of least import in this respect.

The necks of the teeth, and the parts marked $c c$ in the diăgram, should be scratched with a fine steel probe or the edge of a penknife, and the amount of whitisli greasy deposit scraped off noted before and after cleaning. Its presence after cleaning is a certain index that the brush and silk have not reached their goal.

A shaving mirror, with a strong electric light let into the lower part, greatly facilitates examination of one's own teeth ; indeed, without a good light such examination is impossible.

Thorough cleansing once daily will suffice to leep the mouth in that state of minimal infection which, perforce we must call healthy. Gingivitis is prevented because the germs are not allowed to stagnate long enough to multiply and infect; caries is prevented since time is not allowed for the weak acids of carbohydrate decomposition to begin their attack on the enamel; tartar is prevented because the first deposited layer is soft and easily rubbed away. If, in spite of all, trouble arise, it will always be found to be in some inaccessible-or, at least, unsearched - spot. That there are such inaccessible spots is true-a partly erupted wisdom tooth is an obvious example-but by far the major portion of dental sepsis is within our control, and some day a septic mouth may bé held as disgraceful as a dirty face. Its only excuse lies in the idea of "salting," an idea to which clinical observation lends no vestige of support-the whole story is one of failing resistance.

For cleaning artificial teeth I know of no soap equal to that supplied by Lacoste et Cie, 70A, Basinghall Street. The brush supplied for use with this soap, though big and ugly, is specially chosen as best adapted to its work by reason of its length and curve and the length of the bristles.

Treatment.

The treatment of dental sepsis, when present, may be summed up shortly: "Clean and render cleansable. The mouth must be'left: in such a state that the patient himself can clean it. : Every filling must be flush with the surrounding tooth and smooth, all tairtar must be removed, every pocket must be destroyed till the denuded root can be thoroughly cleaned. Mère arrest of the flow of pus and removal of tartâr and putrid matter cannot be accounted as cure. Reaccumulation is only a matter of time, and suppuration will fóllow again. The details of treatment and the importancè tô be attached to the degree of sepsis necessarily vary with the patient. "It may be noted first that infection is microscopic, and it may be asked why, in the mouth 'only, it must become macroscopic and tangible before deserving recognition. Resistance is an unknown and varying quantity, and can only be measured by, ofttimes sad, experience. Prevention is often a thankless task, since there is no knowing what has been prevented, bút we know enough of the results of dental sepsis to make it clear that its earliest sign calls for action. The smallest degree must

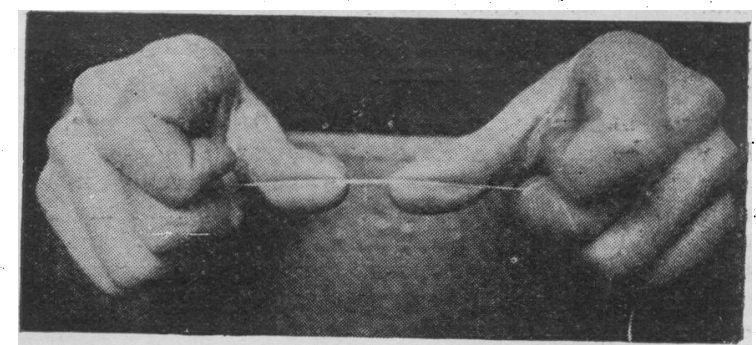

Fig. 16.-Showing how to manipulate the silk thread with the thumbs.

be treated; if left alone, the patient will lose teeth or lealth, or both. ,The treatment of the severer degrees must be decided by; the condition of the patient. : The presence or absence of teeth decides the presence or absence of sepsis. Wlien the patient's health is at stâke, the teeth must go. An eye for an eye and a tooth for a tooth may pass, but an eye for a tooth, a joint for a tooth, a life for a tooth ! If the teeth "are but suspected in these cases they should be dealt with. Unfortunately, of all operations the medical profession appears most to dread extraction of teeth. A man who will assert that the patient is too ill to bear extraction of teeth will at the same time urge exploratory laparotomy. Two things seem to be at the back of this' attitude-a failure to grasp the extent and danger of dental sepsis; and an exaggerated dread of the edentulous state. The extent of dental sepsis is a demonstrable fact, and its danger the logical deduction from modern pathology-a deduction which is daily upheld when put to the test. Daily experience also shows that the edentulous state has practically no pathogenic properties. Especially in hospital practice this is a very important point. If those who are incredulous will inquire, I am sure they will soon discover for themselves a surprising number of healthy and contented edentulous persons. When in severer cases the patient's general condition is yet sound, local measures designed to render every tooth easily cleansable may be adopted, and the patient should be warned that he had better be without teeth he cannot clean.

Cases may be considered "severer" when the teeth are loose or springy in their sockets, when the pockets are deep, when there is visible pus, when there is inflam. matory swelling or hypertrophy of the gum or bone, and when there is pyorrhoea abscess. 
* Very loose teeth, teeth denuded to a root-apex, teeth between whose roots pocketing has extended are beyond treatment. Teeth springy in their sockets seidom recover, and teeth that have been affected with pyorrhoea abscess seldom remain healthy for long, since at the time of the abscess the root is generally denuded to its apex.

\section{VAccines.}

My experience of the vaccine treatment of "pyorrhoea alveolaris" is not such as to lead me to recommend it. We must remember first that the tissue changes ranked under this name are but one part of dental sepsis. If one could be sure of permanently immunizing the tissues of the whole body against all the infecting germs, we should yet have to deal with decomposition of stagnant débris. No amount of vaccines can prevent reaccumula. tion or decomposition of débris. Unfortunately, my experience is that vaccines confer at best but a transient immunity against the organisms of dental sepsis. I have had to extract teeth for acute pyorrhoea abscess immediately after a prolonged course of vaccine treatment. Time is beginning to show us the value of the vaccine treatment of this condition, and the verdict seems to me unfavourable. Within a short period I have had to deal radically with two cases which had undergone vaccine treatment; both the local and general symptoms demanded it. As a broad rule of practice when complete extirpation of the teeth is contemplated, I always consider the question of first preparing a vaccine in case it be needed later for the treatment of secondary conditions, such as joint infections; otherwise I am content to treat without vaccines. The use of vaccines is recommended by some as a preliminary to extensive extraction. Neither in private nor hospital practice have I found this necessary, and it seems to me unwise to prolong the patient's risks of infection during the time required for vaccine treatment.

The Clinical Significance of Dental Sepsis.

It will be seen that $I$ am of those who regard the recognition of dental sepsis as revolutionary in the practice of medicine and surgery. I claim that my faith is grounded on demonstrable fact.

$I$ spend a large part of my spare time in examining skulls and noting the evidences of dental sepsis. A the same time I ascertain as minutely as possible the feeding habits of the subjects, and my deduction is that the major part of dental sepsis is the result of eating soft, sticky, starchy, and sugary food, the word "sticky" being the most important. Of the minor part some is due to stagnation and multiplication of germs with little or no food stagnation, some to gross injury in eating, and some to the results of excessive grinding (too coarse meal).

WALKER AND SELLARDs have undertaken (Philippine Journal of Science, Section B, Tropical Medicine, vol. viii, No. 4) a very interesting study of the relationship of different species of amoebae to tropical dysentery. They made 60 feeding experiments with different species of amoeba and entamoeba which have been implicated in the production of this disease. The experiments differ from others previously carried out in that the number of com. parative tests made of different species was large; that the experiments were carefully controlled, the species of amoeba fed to the individual being recovered; and that the experiments were carried out on men. It was found that cultivable amoebae-amoebae from water, stools of healthy persons, and other non-parasitic sources-were incapable of living parasitically in the intestinal track of man. On the other hand, Entamoeba coli can and does live in the intestine, but it is non-pathogenic, and plays no part in the production of dysentery. It cannot be cultivated outside the body on Musgrave and Clegg's medium. Of 18 men who took Entamoeba histolytica as an experi ment, 4 , or 22.2 per cent., up to the time this paper was written, had developed entamoebic dysentery, the incubation period in these cases being twenty, ninety-five, eightyseven, and flfty-seven days. The patients infected were those who had ingested the entamoeba from normal stools of carriers. The authors conclude that $E$. histolytica and $E$. tetragena are the same, that they are the essential cause of entamoebic dysentery, and that they are proper parasites, and cannot be cultivated outside the body. In Nature it would appear as if the infection was spread from man to man chiefly or solely by the carriers-that is persons harbouring resisting or encysted stages of the parasite in their stools.

\section{THE BRITISH MEDICAL ASSOCIATION AND THE MEDICAL PROFESSION.}

\section{A CRITICAL REVIEW OF THEIR RELATIONS.}

\author{
(Continued from page 1253.)
}

III.

POOR LAW MEDICAL OFFICERS.

OF the innumerable battles that the British Medical Association has fought on behalf either of the medical profession as a whole, or of sections, or of individual members thereof, the very first was for the protection of Poor Law medical officers. It was a war, however, rather than a battle, for, not content with its early successes, the Association expended energy in this direction for many years in succession. It continued to do so even after it had brought into existence the affiliated but independent body specially devoted to the interests of Poor Law medical officers. Indeed, when need be, it continues to wage war through its local Divisions, its central organiza. tion, and its Journal at the present time.

So large a proportion of all medical men engage in Poor Law practice at one time or another of their careers as to lend this particular struggle a special interest of its own. Apart from this, many of its incidents and the general trend of events are so typical of the general character of all the campaigns conducted by the Association as to make it worth while to devote rather more space to their description than will be possible when speaking of what the Association has done for other branches of medical work. Were it worth while to recount the various incidents in detail, the fashion of the drafting of some of the reports which were issued at various times, and some of the accounts of the meetings that took place, would be found to have a curiously twentieth century flavour about them. In fact, the existing records of one particular debate offer the fullest support for those who like to apply to medical mankind the old French proverb, Plus ça change, plus c'est la même chose.

Just about the time that the Association first came into existence a new Poor Lair Act had been passed. Practically it put any medical man who undertook to do work for the poor in his neighbourhood absolutely at the mercy of boards of guardians and of certain Government officers known as Poor Law Commissioners, and to the hardships and various evils that arose from this position of affairs the Council of the Association drew attention at the third annual meeting of the Association in 1835. In addition to describing the position of matters this report stated that the Association had already addressed the Poor Law Commissioners on the subject, but had obtained none but evasive replies; and finally, after it had been indicated verbally that the Royal College of Physicians, the Royal College of Surgeons, and the Society of Apothecaries all took up a non possumus attitude in the matter, the meeting adopted a recommendation that a special committee should be appointed to investigate matters in detail, in order that the Government might again be attacked by the Council with a direct mandate from the Association at its back.

This committee duly reported in the following year, and at the General Meeting of the Association in 1837 it was decided to submit the report to Parliament, together with a memorial, praying that effect might be given to the suggestions contained. Copies were also ordered to be sent to the two colleges and the Society of Apothecaries, again inviting their co-operation, and letters; including copies of the report, were sent to medical men resident in every part of the kingdom, with the idea of securing as much publicity as possible and of securing the influence of members of Parliament by personal interviews.

The report was a very able docunent, which considered in detail the general principles that should underlie the provision of medical attendance on the less wealthy classes, and took a view of the work of a Poor Law medical officer which if true to-day was even truer still at the date that it was written:

The offlce of parish surgeon should combine the highest qualiflcations of the medical body, it being clear that no professional responsibility whatever, public or private, equal it in variety and extent. It embraces and presents, in daily profusion, cases in medicine, 